\title{
A Molecular Link between miRISCs and Deadenylases Provides New Insight into the Mechanism of Gene Silencing by MicroRNAs
}

\author{
Joerg E. Braun, Eric Huntzinger, and Elisa Izaurralde \\ Department of Biochemistry, Max Planck Institute for Developmental Biology, Spemannstrasse 35, \\ 72076 Tübingen, Germany \\ Correspondence: elisa.izaurralde@tuebingen.mpg.de
}

MicroRNAs (miRNAs) are a large family of endogenous noncoding RNAs that, together with the Argonaute family of proteins (AGOs), silence the expression of complementary mRNA targets posttranscriptionally. Perfectly complementary targets are cleaved within the basepaired region by catalytically active AGOs. In the case of partially complementary targets, however, AGOs are insufficient for silencing and need to recruit a protein of the GW182 family. GW182 proteins induce translational repression, mRNA deadenylation and exonucleolytic target degradation. Recent work has revealed a direct molecular link between GW182 proteins and cellular deadenylase complexes. These findings shed light on how miRNAs bring about target mRNA degradation and promise to further our understanding of the mechanism of miRNA-mediated repression.

Mising icroRNAs (miRNAs) are genome-encoded, 21-23-nucleotide noncoding RNAs that posttranscriptionally silence mRNA targets containing complementary sequences (Bartel 2009). To exert their regulatory functions, miRNAs associate with Argonaute (AGO) proteins in effector complexes known as miRNA-induced silencing complexes (miRISCs). These complexes promote endonucleolytic cleavage of fully complementary targets or translational repression, mRNA deadenylation, and exonucleolytic decay of targets with partial complementarity (see Figs. 1 and 2 and Bartel 2009 for a detailed description of miRNA-target recognition; Djuranovic et al. 2011; Huntzinger and Izaurralde 2011). Invertebrate genomes contain at least 100 miRNA genes, whereas vertebrate and plant genomes possess 500 to 1000 miRNA genes (Bartel 2009; Voinnet 2009). Computational predictions and functional studies indicate that the highly expressed miRNAs can potentially regulate hundreds of different mRNAs, suggesting that a significant proportion of eukaryotic transcriptomes ( $~ 50 \%$ in humans) is subject to miRNA regulation (Bartel 2009; Voinnet 2009).

Given the large number of potential targets, it is not surprising that miRNAs play roles in nearly all developmental and cellular processes investigated thus far (Sayed and Abdellatif 2011). It is clear that changes in miRNA expression levels are associated with many human

Editors: John W.B. Hershey, Nahum Sonenberg, and Michael B. Mathews

Additional Perspectives on Protein Synthesis and Translational Control available at www.cshperspectives.org

Copyright (C) 2012 Cold Spring Harbor Laboratory Press; all rights reserved; doi: 10.1101/cshperspect.a012328

Cite this article as Cold Spring Harb Perspect Biol 2012;4:a012328 
J.E. Braun et al.

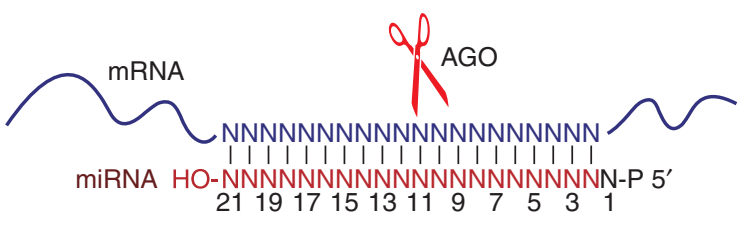

Figure 1. Mechanism of miRNA-mediated gene silencing (fully or nearly complementary targets). A miRNA bound to an AGO protein recognizes mRNA targets containing fully or nearly complementary binding sites. In plants, these binding sites are predominantly located within the ORF. The AGO protein cleaves the mRNAwithin the base-paired region (between nucleotides opposite to nucleotides 10 and 11 of the miRNA strand, which should be base-paired for AGO to cleave). Following cleavage, the mRNA decay intermediates are degraded from the newly generated $3^{\prime}$ and $5^{\prime}$ ends by the exosome and XRN1 or its plant ortholog XRN4 (not shown; Voinnet 2009). The miRNA $5^{\prime}$ terminal nucleotide (shown in black) is buried in the $5^{\prime}$-phosphate binding pocket of AGOs and is not available for pairing with the target.

diseases, such as cancer and metabolic disorders (Esteller 2011; Sayed and Abdellatif 2011).

During the past decade, remarkable progress has been made in understanding miRNA biogenesis and function (Krol et al. 2010; Esteller 2011; Sayed and Abdellatif 2011); however, the mechanisms by which miRNAs regulate gene expression remain unclear and are still a source of scientific debate (Djuranovic et al. 2011; Huntzinger and Izaurralde 2011). In this review, we focus on the effector step of silencing, that is, on what happens after a mRNA target is recognized by miRISCs. First, the emerging model of the molecular mechanism used by miRNAs to silence mRNA targets is described.

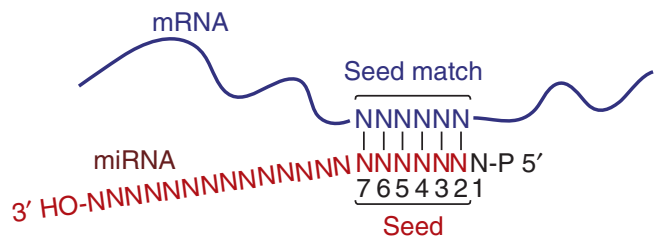

Figure 2. miRNA target recognition in animals. In animals, miRNAs typically recognize partially complementary binding sites, which are generally located in $3^{\prime}$ UTRs. Complementarity to the miRNA "seed" sequence, containing nucleotides $2-7$ or $2-8$, is a major determinant in target recognition and is sufficient to trigger silencing. In some cases, complementarity to the $3^{\prime}$ region of the miRNA might contribute to target binding (not shown, see Bartel 2009). However, even for these sites, miRNA nucleotides 9-12 are generally not complementary to the target, preventing endonucleolytic cleavage by AGOs. In these cases, AGOs recruit a protein of the GW182 family (see Fig. 3).
We then evaluate evidence supporting this model and discuss some key questions that remain to be answered, particularly regarding the mechanistic connections between miRNA-mediated translation repression and mRNA degradation. Studies on the GW182 family proteins, which are key components of miRISCs in animals, have led to many insights into the biochemical mechanisms of silencing. Therefore, in this review, we focus on recent data that have deepened our understanding of how this protein family cooperates with AGOs and cellular factors to bring about silencing.

\section{EMERGING MODEL OF miRNA-MEDIATED GENE SILENCING}

Accumulated data in the miRNA field suggest a model of silencing that begins with the recognition of the target by a miRNA in complex with an AGO protein. In instances when the complementarity between the target and the miRNA is extensive and the AGO protein is catalytically active, the target is cleaved by AGO within the base-paired region (between nucleotides 10 and 11, opposite the miRNA strand) (Fig. 1) (Bartel 2009; Jínek and Doudna 2009; Voinnet 2009). This mechanism appears to be most prominent in plants where miRNAs recognize fully or nearly complementary binding sites, which are generally located in the mRNA open reading frames (ORFs) (Voinnet 2009).

In animals, miRNAs recognize partially complementary binding sites, which are generally 
located in the mRNA $3^{\prime}$ untranslated region (UTR) (Bartel 2009). Complementarity to the 5 ' end of the miRNA — the so-called "seed" sequence - is a major determinant in target recognition and is sufficient to trigger silencing (Fig. 2) (Bartel 2009). Even when target complementarity is not limited to the seed sequence, miRNA nucleotides 9-12 are generally not complementary to the target in animals, preventing endonucleolytic cleavage by AGOs (Bartel 2009; Jínek and Doudna 2009). This is important because in these cases, the AGO proteins are insufficient to mediate silencing and require interaction with additional proteins, including members of the GW182 family (Huntzinger and Izaurralde 2011).

Recruitment of a GW182 protein to a miRNA target triggers translational repression and mRNA deadenylation (Fig. 3). The mechanism of translational repression has yet to be elucidated, al- though increasing evidence points to an inhibition of translation initiation (Djuranovic et al. 2011; Huntzinger and Izaurralde 2011). In contrast, much is known about the mechanism of miRNA-mediated mRNA deadenylation. It is known that deadenylation is performed by the sequential action of two cytoplasmic deadenylase complexes (the PAN2-PAN3 and the CCR4NOT complex) (Fig. 3) (Behm-Ansmant et al. 2006; Chen et al. 2009; Eulalio et al. 2009a; Piao et al. 2010). Both complexes directly interact with GW182 proteins (Braun et al. 2011; Chekulaeva et al. 2011; Fabian et al. 2011).

Depending on the cell type and/or specific target, the deadenylated mRNA target can be stored in a translationally repressed state, as observed, for example, in Caenorhabditis elegans embryos and in cell free extracts that recapitulate silencing (Mathonnet et al. 2007; Thermann and Hentze 2007; Wakiyama et al. 2007; Iwasaki

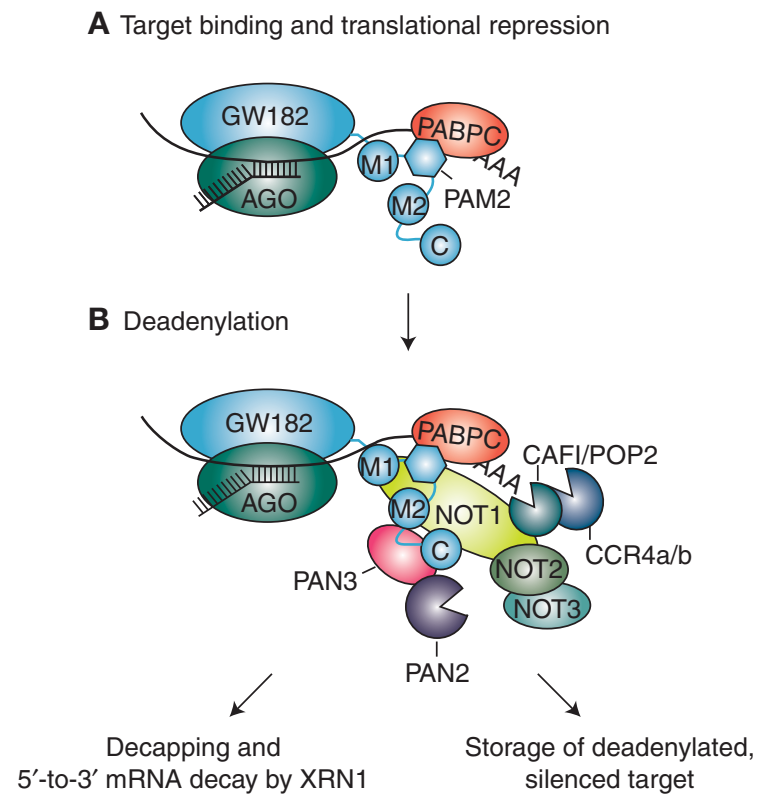

Figure 3. Mechanism of miRNA-mediated gene silencing in animals (partially complementary targets). $(A, B)$ The AGO-GW182 complex represses translation through an unknown mechanism and directs the mRNA to deadenylation. Human GW182 proteins interact with PABPC through the PAM2 motif, with PAN3 through the M2 and carboxy-terminal (C-term) regions and with NOT1 through the M1, M2, and C-term regions. Although translational repression and deadenylation are shown as consecutive steps, the order of events remains controversial, and it is unclear whether the two processes are linked or independent. Depending on the cell type and/or specific target, deadenylated mRNAs can be stored in a deadenylated, translationally repressed state. In animal cell cultures, deadenylated mRNAs are generally decapped and rapidly degraded by the major $5^{\prime}$-to- $3^{\prime}$ exonuclease XRN1. 
J.E. Braun et al.

et al. 2009; Zdanowicz et al. 2009; Wu et al. 2010). However, in diverse organisms and cell types, a reduction in mRNA levels is observed (Bagga et al. 2005; Lim et al. 2005; Wu and Belasco 2005; Behm-Ansmant et al. 2006; Giraldez et al. 2006; Mishima et al. 2006; Rehwinkel et al. 2006; Schmitter et al. 2006; Wu et al. 2006). This can be explained by the observation that deadenylated mRNAs are in generally unstable and are rapidly decapped and degraded by the major 5'-to-3' exonuclease XRN1. Thus, miRNAs accelerate target destruction by recruiting the enzymes involved in one of the two major cellular mRNA decay pathways, namely the $5^{\prime}$-to- $3^{\prime}$ mRNA decay pathway (Rehwinkel et al. 2005; Behm-Ansmant et al. 2006; Eulalio et al. 2007a, 2009a; Chen et al. 2009; Piao et al. 2010). In this pathway, mRNAs are first deadenylated, then decapped and, finally, exonucleolytically degraded from the $5^{\prime}$-end by XRN1.

Although it is now clear how miRNAs cause target degradation, there is more to the story that needs to be understood. For example, GW182 proteins interact with the cytoplasmic poly(A)binding protein (PABPC, where $\mathrm{C}$ stands for cytoplasmic), but it is not known how this interaction contributes to silencing (Fabian et al. 2009; Zekri et al. 2009; Huntzinger et al. 2010; Jínek et al. 2010; Kozlov et al. 2010). Another key question that remains is whether translational repression and deadenylation are interconnected or represent two independent mechanisms used by miRNAs to silence mRNA targets. Finally, understanding how translational repression is achieved remains a major challenge for future studies.

\section{THE GW182 PROTEIN FAMILY: DOMAIN ORGANIZATION}

GW182 proteins were first identified as antigens recognized by serum from a patient with motor and sensory neuropathy (Eystathioy et al. 2002). Their role in the miRNA pathway was revealed in subsequent studies showing that GW182 proteins copurify with AGO-containing complexes and their depletion inhibits silencing of miRNA targets (Jakymiw et al. 2005; Liu et al. 2005; Meister et al. 2005; Rehwinkel et al. 2005). In independent studies, the proteins were identified based on mutant phenotypes that reflect the inactivation of the miRNA pathway in C. elegans or in RNAi screens for suppressors of silencing in Drosophila melanogaster (Ding et al. 2005; Behm-Ansmant et al.2006). Subsequently, it was shown that GW182 proteins act downstream of AGOs, in the effector step of silencing (Eulalio et al.2008). In vertebrates and several invertebrate species, there are up to three GW182 paralogs with partially redundant functions (also known as TNRC6A, B and C), whereas there is only one orthologous protein in D. melanogaster (Dm GW182).

GW182 proteins typically contain an amino-terminal region (N-term) with multiple glycine-tryptophan $(\mathrm{GW})$ repeats (i.e., GW, WG, or GWG), a central ubiquitin-associated (UBA) domain, and an RNA recognition motif (RRM) (Fig. 4) (Eulalio et al. 2009b). Additional regions of the protein include a glutamine-rich (Q-rich) region, which is located between the UBA domain and the RRM, and middle (Mid) and carboxy-terminal (C-term) regions containing fewer or no GW repeats (Fig. 4) (Eulalio et al. 2009b). Interestingly, this domain organization is not conserved in the C. elegans proteins AIN-1 and AIN-2, which are highly divergent members of the protein family (Ding et al. 2005; Zhang et al. 2007; Eulalio et al. 2009b).

A fascinating aspect of the GW182 family is that the protein regions that are functionally relevant for silencing are predicted to be disordered. For example, the N-term GW repeat region of the proteins mediates binding to the AGO proteins and thus is essential for miRNA-mediated gene silencing (Behm-Ansmant et al. 2006; Till et al. 2007; Eulalio et al. 2008, 2009c; Lazzaretti et al. 2009; Lian et al. 2009; Takimoto et al. 2009). This region is predicted to be unstructured and interacts with AGOs through multiple GW repeats, which contribute to the interaction in an additive manner. The exact location and number of repeats are, however, not conserved.

The Mid and C-term regions (collectively termed the silencing domain), both predicted to be disordered, are also essential for silencing (Chekulaeva et al. 2009; Eulalio et al. 2009c; Lazzaretti et al. 2009; Zipprich et al. 2009; 


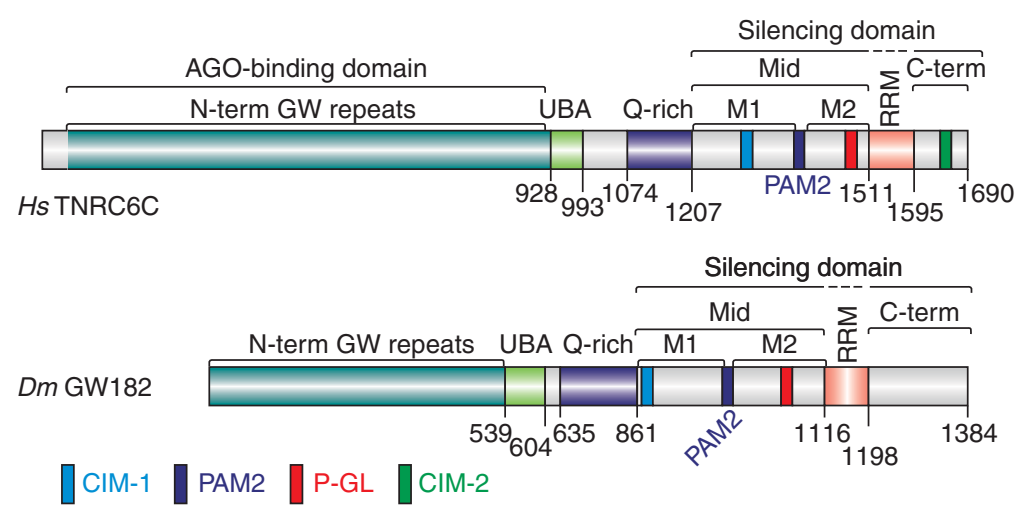

Figure 4. GW182 protein family. Domain organization of GW182 proteins. The amino-terminal AGO-binding domains contain multiple GW repeats (not shown). The silencing domain includes the Mid and carboxyterminal regions but not the RRM, which is dispensable for silencing. The Mid and C-term regions contain a variable number of GW repeats (not shown) and additional motifs termed CIM-1, CIM-2, and P-GL. Human TNRC6C and Dm GW182 are shown as representative family members of the GW182 proteins. Abbreviations: UBA, ubiquitin-associated domain; Q-rich, region rich in glutamine; Mid, middle domain with PAM2 motif (dark blue), which divides the Mid region into the M1 and M2 regions; RRM, RNA recognition motif; C-term, carboxy-terminal region; $\mathrm{N}$-term, amino-terminal.

Huntzinger et al. 2010). For instance, GW182 protein mutants lacking the silencing domain generally do not rescue silencing in cells depleted of endogenous GW182, even though these proteins interact with AGOs and are active in tethering assays (Chekulaeva et al. 2009; Eulalio et al. 2009c; Zekri et al. 2009; Huntzinger et al. 2010; Braun et al. 2011). Recent work has shown that the silencing domains of human GW182 proteins provide a binding platform for PABPC and subunits of the two major cytoplasmic deadenylase complexes (the PAN2-PAN3 and CCR4-NOT complexes) (Fabian et al. 2009, 2011; Zekri et al. 2009; Huntzinger et al. 2010; Jínek et al. 2010; Kozlov et al. 2010; Braun et al. 2011; Chekulaeva et al. 2011). We discuss the contribution of these interactions to miRNAmediated repression below.

\section{THE GW182 PROTEIN FAMILY: INTERACTION NETWORK}

GW182 Proteins Are PABP-Interacting Proteins (Paips)

The interaction of GW182 proteins with PABPC is mediated by a conserved PAM2 motif (PABPbinding motif 2) located in the Mid region
(Fig. 4) (Fabian et al. 2009; Huntzinger et al. 2010; Jínek et al. 2010; Kozlov et al. 2010). The PAM2 motif was first identified in the translational regulators Paip1 and Paip2 (PABP-interacting proteins 1 and 2) (Derry et al. 2006). This motif confers direct binding to the PABPC carboxy-terminal domain termed the MLLE domain (Fabian et al. 2009; Huntzinger et al. 2010; Jínek et al. 2010; Kozlov et al. 2010). Moreover, GW182 protein sequences downstream of the PAM2 motif (termed M2) together with the C-term region mediate indirect binding to PABPC in vivo (Huntzinger et al. 2010). Although indirect, this interaction is dominant over that mediated by the PAM2 motif in D. melanogaster cells (Zekri et al. 2009; Huntzinger et al. 2010).

Interestingly, in addition to PABPC, the PAM2 motif of mammalian GW182 proteins interacts with the EDD protein (also known as hyperplastic discs [HYD]), which like PABPC, contains an MLLE domain (Su et al. 2011). Because EDD also interacts indirectly with decapping factors and the CCR4-NOT complex (Su et al. 2011), it could help to recruit these factors to miRNA targets. The extent of the contribution of EDD to silencing and the 
J.E. Braun et al.

conservation of its role in other species remains to be established. Furthermore, because PABPC and EDD interact with GW182 proteins in a mutually exclusive manner, it will be important to determine whether EDD and PABPC play redundant or distinct roles in silencing, for example, leading to different outcomes.

\section{GW182 Proteins Interact Directly with Deadenylase Complexes}

In addition to PABPC, the silencing domains of human GW182 proteins confer direct binding to PAN3 and NOT1, which are subunits of the PAN2-PAN3 and CCR4-NOT deadenylase complexes, respectively (Braun et al. 2011; Chekulaeva et al. 2011; Fabian et al. 2011). Binding to PAN3 requires the $\mathrm{M} 2$ and $\mathrm{C}$-term regions of the silencing domain (Braun et al. 2011), whereas NOT1 binding is mediated by the M1, M2, and C-term regions (Fig. 4), which contribute to the interaction in an additive manner (Braun et al. 2011; Chekulaeva et al. 2011; Fabian et al. 2011).

A precise mapping of the interaction with the CCR4-NOT complex identified two distinct short linear motifs within the M1 and C-term regions of human GW182 silencing domains. These motifs were termed CCR4 interaction motifs (CIM)-1 and 2, respectively (Fig. 4) (Fabian et al. 2011). CIM-2 is characterized by a LWG repeat, suggesting that GWrepeats can mediate interactions with protein partners other than AGOs, depending on the context. Accordingly, alanine substitutions of all tryptophan residues in the M2 and C-term regions of the human TNRC6C silencing domain abolished both CCR4-NOT and PAN2PAN3 binding (Chekulaeva et al. 2011).

Interestingly, although CIM-1 and CIM-2 recruit the CCR4-NOT complex to mRNA targets, only CIM-2 supports full deadenylation, suggesting that these motifs are functionally distinct (Fabian et al. 2011). These findings also indicate that recruitment of the CCR4-NOT complex is not sufficient to promote processive deadenylation and that an "activation step" is involved. The CIM-2 motif, but not CIM-1, can mediate this activation. This opens the perspec- tive that GW182 proteins do not merely recruit the CCR4-NOT complex but enhance deadenylation rates (Fabian et al. 2011).

The interactions with the deadenylase complexes are conserved in D. melanogaster (Braun et al. 2011; Chekulaeva et al. 2011); however, the mode of interaction is not. For instance, only the CIM-1 motif is present in Dm GW182, whereas CIM-2 is absent (Fig. 4) (Fabian et al. 2011). Furthermore, in contrast to human GW182s, deletion of the silencing domain in Dm GW182 does not abolish binding to NOT1 (Braun et al. 2011; Chekulaeva et al. 2011), suggesting that additional binding motifs, upstream of the silencing domain, are present in Dm GW182.

\section{GW182 Proteins Contain a Conserved Proline-Rich Motif}

A recent study using zebrafish led to the identification of a proline rich motif (P-GL motif) (Fig. 4) in the M2 region of the silencing domains that, together with the PAM2 motif, contributes to translational repression and the deadenylation of polyadenylated targets, as well as to translational repression in the absence of deadenylation (i.e., targets lacking a poly(A) tail) (Mishima et al. 2012). Interestingly, this motif is not required for PABPC or deadenylase binding. This finding adds another layer of complexity to GW182 protein function, as it suggests that the proteins interact with another, yet unidentified partner. The P-GL motif is conserved in vertebrate and insect GW182 proteins; thus, it would be of interest to identify its potential binding partners.

\section{Plasticity of the GW182 Protein Interaction Network}

The study of the GW182 protein interaction network in different species has revealed that these proteins interact with their partners (e.g., AGOs, PABPC, PAN3, and NOT1) through short linear motifs (SLiMs) (Davey et al. 2012) embedded in unstructured regions. This provides one explanation for why the binding of GW 182 proteins to their partners requires multiple and partially redundant motifs (e.g., multiple GW repeats), 
as SLiMs provide low-affinity binding and multiple motifs are required for high-affinity (avidity) interactions (Davey et al. 2012). This also explains why the partners are conserved but the molecular details of the interaction change throughout evolution (e.g., Dm GW182 lacks CIM-2). Indeed, linear motifs are evolutionarily plastic, as only a small number of point mutations in a disordered region of a protein sequence can result in a gain, loss, or relocation of SLiMs. In this context, it is interesting to note that the C. elegans GW182 protein AIN-1 interacts with PABPC, PAN3, NOT1, and NOT2, despite that it lacks all the motifs described above (KuzuogluÖztürk et al. 2012). Therefore, it is possible that AIN-1 has evolved different ways to interact with the same partners.

\section{ROLE OF PABPC}

The PAM2 motif is highly conserved among vertebrate and insect GW182 proteins, and the interaction with PABPC is well documented by biochemical and structural studies (Fabian et al. 2009; Zekri et al. 2009; Huntzinger et al. 2010; Jínek et al. 2010; Kozlov et al. 2010). However, little is known regarding how the GW182PABPC interaction contributes to silencing. Several mechanisms have been proposed, which are not mutually exclusive.

The first model is that the interaction of GW182 proteins with PABPC interferes with mRNA circularization (Fabian et al. 2009; Zekri et al. 2009). It is known that PABPC (bound to the mRNA poly(A) tail) interacts with eIF4G (associated with the cap structure through interaction with the cap-binding protein eIF4E), giving rise to circular mRNAs that are efficiently translated and protected from degradation (Derry et al. 2006). By analogy with Paip2, GW182 proteins may compete with eIF4G for binding to PABPC, thereby preventing mRNA circularization and consequently inhibiting translation.

The second possibility is that the PABPCGW182 interaction reduces the affinity of PABPC for the poly(A) tail, as described for Paip2 (Derry et al. 2006). The third model is that the PABPC-GW182 interaction acceler- ates miRNA-mediated deadenylation. This last model is supported by the observation that in cell-free extracts from mouse Krebs-2 ascites cells, PABPC depletion or mutations in the PAM2 motif of TNRC6C reduce the rate, but not the extent, of deadenylation (Fabian et al. 2009, 2011; Jínek et al. 2010).

It is also unclear whether PABPC is required for silencing, as conflicting lines of evidence have been reported (Fabian et al. 2009; Zekri et al. 2009; Huntzinger et al. 2010; Jínek et al. 2010; Braun et al. 2011; Fukaya and Tomari, 2011; Mishima et al. 2012). A role for PABPC in silencing is supported by three observations: (1) overexpressing PABPC or an excess of Paip2PAM2 peptide reduces silencing under conditions in which general translation is not affected (Fabian et al. 2009; Zekri et al. 2009; Walters et al. 2010); (2) depletion of PABPC from Krebs-2 ascites cell extracts inhibits miRNA-mediated deadenylation (Fabian et al. 2009, 2011; Jínek et al. 2010); and (3) in complementation assays, human GW182 mutants that do not interact with PABPC (i.e., carrying mutations in the PAM2 motif) are impaired in rescuing silencing in cells depleted of the endogenous GW182 proteins (Huntzinger et al. 2010; Braun et al. 2011). These results indicate that the PABPC-GW182 interaction is important, although not essential, for silencing in these cells. It has also been shown that PABPC-binding is not sufficient for silencing in vivo (Huntzinger et al. 2010; Braun et al. 2011) or for GW182-mediated deadenylation in vitro (Fabian et al. 2011).

Arguing against a role for PABPC in silencing is the observation that mRNAs lacking a poly(A) tail (that is, they cannot circularize and are not deadenylated) are nevertheless silenced (Humphreys et al. 2005; Pillai et al. 2005; Wu et al. 2006; Eulalio et al. 2008, 2009a; Chekulaeva et al. 2011). This suggests that PABPC is either not required for silencing or is only required for repression of polyadenylated targets. Alternatively, PABPC could be recruited to unadenylated targets through interactions with translation factors and bind to GW182 in the absence of a poly(A) tail, but how this interaction might contribute to silencing is currently unknown. 
J.E. Braun et al.

Remarkably, in tethering assays, multiple and nonoverlapping fragments of $\mathrm{Dm}$ GW182, including amino-terminal fragments that do not interact with PABPC, trigger translational repression and mRNA degradation (Chekulaeva et al. 2009; Fukuya and Tomari 2011; Yao et al. 2011). These results were interpreted as evidence that interactions of GW182 proteins with PABPC are not required for silencing ( Fukaya and Tomari 2011). There are alternative explanations for this observation, including that tethering assays may not recapitulate all steps of silencing. Indeed, in complementation assays, amino-terminal fragments of GW182 (lacking the silencing domain) fail to complement the silencing of a large number of targets (Eulalio et al. 2009c). Thus, although tethering assays are an invaluable tool in the dissection of the role of GW182 protein domains in silencing, conclusions from these assays should be validated in complementation assays.

Fukaya and Tomari (2011) also observed silencing in extracts in which PABPC was inhibited from functioning in translation by the addition of an excess of Paip2, which displaces PABPC from the mRNA poly $(\mathrm{A})$ tail and competes with GW182 for binding to PABPC. Based on these observations, they concluded that PABPC is dispensable for silencing. Yet, if the role of the GW182-PABPC interaction were to facilitate $\mathrm{PABPC}$ dissociation from the poly $(\mathrm{A})$ tail, then this interaction would become dispensable in extracts in which PABPC has been removed from the poly(A) tail by Paip 2 .

The most compelling evidence supporting a nonessential role for PABPC is that its depletion does not affect translational repression or target degradation in zebrafish embryos (Mishima et al. 2012). Paradoxically, in this system, simultaneous mutations in the PAM2 and P-GL motifs of zebrafish GW182 (TNRC6A) strongly impaired the silencing activity of the protein in tethering assays.

A potential explanation for the disparate observations outlined above is that PABPC might be required for silencing of specific targets and/or under specific cellular conditions (see below). This possibility, together with alternative roles for PABPC in silencing, need to be explored in future studies. Until the molecular mechanism of silencing is fully understood, PABPC involvement cannot be ruled out.

\section{ROLE OF CYTOPLASMIC DEADENYLASE COMPLEXES}

In eukaryotes, the mRNA poly(A) tail is removed by the sequential action of two cytoplasmic deadenylase complexes (Chen et al. 2011). The dimeric PAN2-PAN3 complex deadenylates mRNAs in a distributive manner and is responsible for shortening long poly(A) tails to $\sim 50-110$ nucleotides, depending on the specific mRNA and organism of study (Chen et al. 2011). The PAN2-PAN3 complex is recruited to mRNA targets via interaction PAN3 with PABPC. PAN3 also recruits PAN2, the catalytic subunit of the complex to mRNA targets (Siddiqui et al. 2007). The second, processive phase of deadenylation is catalyzed by the CCR4-NOT complex, which can rescue cytoplasmic mRNA deadenylation in the absence of the PAN2-PAN3 complex (Chen et al. 2011). The conserved core of the metazoan CCR4NOT complex consists of five subunits: NOT1, NOT2, NOT3 (also known as NOT3/5), and two catalytically active subunits, CCR4a or its paralog CCR4b and CAF1 or its paralog POP2 (Fig. 3) (Chen et al. 2011).

The relative roles of the two deadenylase complexes in the miRNA pathway are well defined. The CCR4-NOT complex provides a major contribution to miRNA target decay, whereas the PAN2-PAN3 complex plays only a minor role. For example, PAN2 depletion does not prevent miRNA target degradation, whereas the depletion of components of the CCR4NOT complex inhibits miRNA target deadenylation and subsequent degradation (BehmAnsmant et al. 2006; Eulalio et al. 2009a; Piao et al. 2010; Braun et al. 2011). Accordingly, overexpression of catalytically inactive CCR4a, CAF1, or POP2 suppresses silencing in a dominant-negative manner in human cells (Chen et al. 2009; Piao et al. 2010). By contrast, although overexpression of a catalytically inactive PAN2 mutant slows the initial phase of miRNA target deadenylation (Chen et al. 2009), it does 
not suppress silencing, supporting the conclusion that the PAN2-PAN3 complex is involved in but is not essential for deadenylation of miRNA targets.

Despite the important role of the deadenylase complexes in the miRNA pathway, until recently, the molecular mechanism underlying their recruitment remained controversial. A translational-repression-only model for miRNA silencing suggested that deadenylases are recruited to miRNA targets by default, as an indirect consequence of a primary inhibitory effect of miRISCs on translation (reviewed by Djuranovic et al. 2011). The new finding that human GW182 proteins interact with PAN3 and NOT1 (Braun et al. 2011; Chekulaeva et al. 2011; Fabian et al. 2011) definitively shows that the silencing machinery recruits these complexes directly and thus that deadenylation is a direct effect of miRNA regulation and not a mere consequence of translational repression.

\section{ROLE OF THE DECAPPING COMPLEX AND DECAPPING ACTIVATORS}

Generally, deadenylated mRNAs are committed to decapping and $5^{\prime}$-to- $3^{\prime}$ exonucleolytic degradation in somatic cells. The decapping enzyme DCP2 requires cofactors for full activity. In metazoa, these include DCP1, EDC4 (also known as Ge-1), Pat, and the DEAD-box protein RCK/ p54. These decapping activators are also involved in miRNA-mediated mRNA destabilization (Huntzinger and Izaurralde 2011). For instance, mRNA levels of predicted and validated miRNA targets increase when decapping activators are depleted or when dominant-negative forms are overexpressed (Rehwinkel et al. 2005; Behm-Ansmant et al. 2006; Eulalio et al. 2007a, 2009a; Chen et al. 2009; Piao et al. 2010). However, target protein levels are not fully restored (Eulalio et al. 2007a). This is consistent with the observation that decapping inhibition causes accumulation of deadenylated decay intermediates (because deadenylation precedes decapping), and these deadenylated mRNAs are not translated efficiently, providing one explanation for why protein levels are not rescued.
One question that remains is whether decapping of miRNA targets occurs as a consequence of deadenylation or whether miRISCs can also recruit components of the decapping machinery independent of deadenylation. For example, RCK/p54 coimmunoprecipitates with AGOs in human cells (Chu and Rana 2006), although it is not known whether this interaction is direct. The next question is whether decapping activators are required for silencing. In cell-free extracts, decapping does not occur even when miRNA targets are deadenylated, indicating that decapping per se is not required for the establishment of silencing in vitro. Nonetheless, decapping activators act as general repressors of translation even in the absence of decapping (Nissan et al. 2010); thus, it would be of interest to determine whether they play a more direct role in translational repression of miRNA targets.

\section{GW182 INTERACTION NETWORK: THE ROLE OF REDUNDANT AND COMBINATORIAL INTERACTIONS}

The information available on the GW182 interaction network reveals some important features, including redundant and combinatorial interactions, which must be taken into account in considering how GW182 proteins exert their repressive functions.

Redundancy is manifested at three different levels. The first level is because of gene duplication events that generated two to three GW182 paralogs in almost all the animal species investigated thus far. These paralogs are, in addition, present in multiple isoforms. Likewise, many species contain multiple AGO and PABPC paralogs. Moreover, two paralogs of each of the catalytic subunits of the CCR4-NOT complex are expressed in vertebrates (CCR4a and CCR4b and CAF1 and POP2), leading to the assembly of at least four CCR4-NOT complexes. Redundancy at the level of the individual silencing factors enables the assembly of multiple alternate miRISCs, which might be functionally distinct, resulting in different outputs.

Another layer of redundancy is observed in the connections between network components. GW182 proteins interact with deadenylases, 
J.E. Braun et al.

PABPC, EDD, and probably other unknown partners. At the same time, PABPC and deadenylases interact with each other. For instance, PAN3 also contains a PAM2 motif and directly interacts with PABPC (Siddiqui et al. 2007), thereby providing an indirect link between GW182 and PABPC (Braun et al. 2011). Furthermore, it has been suggested that the two deadenylase complexes interact and are part of a larger multiprotein complex in vivo (Chen et al. 2011). Finally, EDD interacts directly with the PAM2 motif of GW182 and indirectly with the decapping activator RCK/p54 and the CCR4NOT complex (Su et al. 2011). These examples imply that GW182 proteins can recruit deadenylases either directly or indirectly through PABPC or EDD and can interact with PABPC directly or indirectly through PAN3. Again, although these examples indicate high redundancy, the functional outcomes of direct or indirect recruitment of partners might differ.

A third layer of redundancy is observed at the level of GW182 protein sequences, which is consistent with the existence of alternative ways to recruit partners mentioned above. For instance, individual deletion of Dm GW182 M1, $\mathrm{M} 2$, or C-term regions or of the PAM2 motif does not abolish silencing, but a combination of two or more deletions reduces or abrogates silencing activity in complementation assays (Eulalio et al. 2009c; Huntzinger et al. 2010; Braun et al. 2011; Chekulaeva et al. 2011).

Additionally, other proteins may influence the composition of GW182 complexes in a target-specific manner. For example, the Puf-9 protein facilitates silencing of miRNA targets by different mechanisms (reviewed by Pasquinelli 2012). Puf proteins interact with the CCR4NOT complex, raising the possibility that for these targets, the interaction of GW182 with the CCR4-NOT complex might become dispensable.

In sum, the assembly of functional miRISC complexes is achieved through redundant and combinatorial interactions among silencing factors. This opens the possibility that depending on the exact combination of these factors and the cellular context (which specifies the relative expression levels of different factors), the func- tional output might differ, providing a potential explanation for the different modes of miRNAmediated regulation reported in the literature. Additionally, depending on the relative levels of all factors in a particular cell or tissue, a specific GW182 partner might become essential or dispensable.

\section{GW182 PROTEINS IN PLANTS}

In general, plant miRNAs guide AGOs to nearly perfectly complementary targets, which are then cleaved by the endonucleolytic activity of the AGO proteins (Voinnet 2009). However, evidence exists that plant miRNAs can also repress translation (Aukerman et al. 2003; Chen et al. 2004; Gandikota et al. 2007; Brodersen et al. 2008; Dugas and Bartel 2008). Until recently, it has remained unclear how much translational control is exerted by miRNAs over the plant proteome and how this repression is achieved, as plants lack GW182 orthologs. A recent report presents evidence that plants contain a functional GW182 analog, termed SUO (Yang et al. 2012).

SUO is a large protein with an amino-terminal bromo-adjacent homology (BAH) and transcription elongation factor S-II (TFS2N) domains (Yang et al. 2012). The only feature SUO shares with GW182 proteins is the presence of two carboxy-terminal GW repeats. In contrast to animal GW182 proteins, SUO is predominantly nuclear, although a small fraction of the protein localizes to cytoplasmic mRNAprocessing bodies (P-bodies), as shown for animal GW182s (Yang et al. 2012). Indeed, in animal cells, GW182 proteins are mainly cytoplasmic but they are also detected in P-bodies, which are cytoplasmic foci where proteins involved in translational repression, mRNA decapping and decay accumulate (Eulalio et al. 2007b). Notably, the functional significance of this localization remains unclear, as GW182 proteins that fail to localize to P-bodies are active in complementation assays (Eulalio et al. 2007c, 2009b).

Evidence that SUO plays a role in miRNAmediated translational repression is based on the phenotype of suo mutants, which is reminiscent of the phenotype observed in plants in 
which the miRNA pathway is impaired (Yang et al. 2012). Additionally, in suo-mutant plants, the expression of miRNA targets that are known to be regulated at the translational level increases without changes in their mRNA levels (Yang et al. 2012). It remains to be seen whether SUO interacts with AGOs or with any of the known GW182 interaction partners (e.g., PABPC and deadenylases) and whether it uses a similar mechanism to repress mRNA targets.

Evidence for similarities in the mechanism of silencing between plants and animals includes the identification of enhancer of decapping-4 (EDC4) in screens for suppressors of miRNA-mediated gene silencing both in $D$. melanogaster cells and Arabidopsis thaliana (Eulalio et al. 2007a; Brodersen et al. 2008). However, the molecular details may differ between these two kingdoms; in D. melanogaster, EDC4 plays a role in miRNA target degradation through deadenylation and decapping, whereas in plants, EDC4 may play a role in translational repression (Brodersen et al. 2008).

The study by Yang et al. (2012) also provides important information regarding the contribution of miRNA-mediated translational repression to silencing in plants, by showing that putative null alleles of suo have a weak phenotype compared to the phenotypes of null alleles of DCL1 (the dicer protein that produces miRNAs in A. thaliana). Thus, translational repression may not be essential for miRNA target silencing in plants. However, at present, we cannot exclude the possibility that plant genomes encode additional GW182 analogs (Yang et al. 2012).

\section{MECHANISMS OF TRANSLATIONAL REPRESSION}

Earlier studies indicated that animal miRNAs predominantly repress translation, and this repression was proposed to occur in four distinct ways: (1) cotranslational protein degradation (Nottrott et al. 2006); (2) inhibition of translation elongation (Olsen and Ambros 1999; Seggerson et al. 2002; Maroney et al. 2006); (3) premature ribosome dissociation (Petersen et al. 2006); and (4) inhibition of translation initiation (Humphreys et al. 2005; Pillai et al. 2005).
Subsequent studies in different cell types and cell-free extracts of diverse origins supported a role for miRNAs in inhibiting translation initiation (Wang et al. 2006; Mathonnet et al. 2007; Thermann and Hentze 2007; Ding and Grosshans 2009; Iwasaki et al. 2009). More recently, ribosome profiling data and translational rate measurements indicate that translational repression occurs predominantly at initiation (Hendrickson et al. 2009; Guo et al. 2010). However, to date, the precise step that is affected remains elusive.

\section{INTERPLAY BETWEEN TRANSLATIONAL REPRESSION AND DEADENYLATION}

An important question that remains unresolved is whether translational repression and deadenylation are linked. Much evidence exists to suggest that deadenylation and subsequent degradation do not require active translation. For example, miRNA-mediated deadenylation and decay can be observed in the presence of translation inhibitors (e.g., cycloheximide or hippuristanol) (Eulalio et al. 2007a; Wakiyama et al. 2007; Fabian et al. 2009). Furthermore, miRNA-target reporters that are poorly translated because of a defective cap structure (ApppG-cap) or the presence of a stable stemloop structure in the $5^{\prime}$ UTR are nevertheless deadenylated and degraded in an miRNA-dependent manner (Mishima et al. 2006; Wu et al. 2006; Wakiyama et al. 2007; Eulalio et al. 2009a). In fact, an open reading frame is dispensable for miRNA-directed deadenylation as short, ApppG-capped RNAs containing miRNA-binding sites and a poly(A) tail can be efficiently deadenylated in cell-free extracts from mouse Krebs-2 ascites cells (Fabian et al. 2009). Finally, miRISCs directly interact with deadenylases via the GW182 proteins (Braun et al. 2011; Chekulaeva et al. 2011; Fabian et al. 2011). Collectively, these results indicate that miRNAs trigger deadenylation and decay directly and independently of the translation status of the mRNA target. Additionally, the observation that the zebrafish protein DAZL relieves miRNA-mediated repression by counteracting deadenylation, indicates that for some 
J.E. Braun et al.

targets deadenylation provides a major contribution to silencing (Takeda et al. 2009).

Conversely, there is compelling evidence that translational repression can occur in the absence of deadenylation. For example, in some cell-free extracts, zebrafish embryos and Drosophila cells, translational repression precedes deadenylation (Mathonnet et al. 2007; Thermann and Hentze 2007; Fabian et al. 2009; Zdanowicz et al. 2009; Bazzini et al. 2012; Djuranovic et al. 2012). Another finding suggesting that translational repression occurs in the absence of deadenylation is that miRNA reporters in which the poly(A) tail is replaced by a histone mRNA stem-loop structure or a self-cleavable ribozyme are still repressed by miRNAs (Wu et al. 2006; Eulalio et al. 2008, 2009a). Similarly, mRNA targets containing a blocked $\operatorname{poly}(\mathrm{A})$ tail (a poly $(\mathrm{A})$ tail followed by an unrelated sequence that prevents deadenylation) are repressed by miRNAs in the absence of deadenylation (Fukaya and Tomari 2011; Bazzini et al. 2012; Mishima et al. 2012). Finally, in NOT1-depleted cells, some miRNA targets remain translationally repressed even though mRNA degradation is inhibited (BehmAnsmant et al. 2006; Braun et al. 2011). These examples show the existence of deadenylationindependent mechanisms of translational repression.

In sum, translational repression and deadenylation can occur independently. Consequently, mRNA targets can be silenced at the level of translation or mRNA deadenylation and degradation or by a combination of both. Nevertheless, translational repression and deadenylation can still be connected and might represent two distinct outcomes of a single molecular mechanism that simultaneously interferes with translation and triggers deadenylation. The relative contributions of these two processes to silencing may then be determined by the repertoire of proteins bound to the mRNA target and/or the cellular context. Furthermore, the order of events may depend on the relative rates of inhibition of translation and mRNA deadenylation, which in turn may also be target dependent, resulting in targets silenced predominantly at the translational or mRNA levels.
What might be this initial triggering mechanism? Although completely speculative at this time, an interesting possibility is that the recruitment of deadenylase complexes to the 3' UTR of miRNA targets triggers both translational repression and deadenylation. This possibility is suggested by the intriguing observation that depletion of PAN3 and NOT1 suppresses the silencing of unadenylated reporters, suggesting that deadenylase complexes could also contribute to translational repression in addition to promoting deadenylation (Braun et al. 2011; Chekulaeva et al. 2011). Furthermore, a catalytically inactive CAF1 mutant induces translational repression of a reporter mRNA to which it is tethered in the absence of deadenylation (Cooke et al. 2010; Chekulaeva et al. 2011). These data suggest that deadenylase complexes could contribute to translational repression independent of their role in deadenylation. Future work should determine whether deadenylase complexes repress translation and by which mechanism(s).

\section{CONCLUDING REMARKS}

miRNAs have evolved two divergent ways of promoting target silencing: AGO-mediated endonucleolytic mRNA cleavage (fully or nearly complementary targets) and GW182-mediated translational repression and deadenylation (which may or may not be followed by mRNA destabilization). Despite the significant progress made in dissecting the functions of GW182 proteins in recent years, there is still much to learn about this protein family, including identifying their full repertoire of interacting partners. This information will help clarify whether translational repression and deadenylation represent independent outcomes triggered by GW182 proteins through interactions with distinct protein partners or are induced by a single molecular mechanism. In this context, the study of the more divergent members of the GW182 protein family (e.g., C. elegans AIN-1 and AIN-2 or A. thaliana SUO) is likely to lead to a much deeper understanding of these issues in the coming years. It is also not known what types of regulation GW182 protein activity is subjected 
to and whether RNA-binding proteins bound to specific targets modulate their functions.

Another important task for future research in the miRNA field will be to understand how translational repression is achieved. We expect that answers to this question will emerge as more studies examine the molecular structures and functions of silencing factors and how they interact to assemble into active effector complexes.

In summary, in recent years significant advances in our understanding of the mechanism of silencing have been made, both in animals and plants. Although the molecular details remain to be elucidated, new findings revealed an unanticipated direct connection to the CCR4NOT complex, which is a master posttranscriptional regulator in eukaryotic cells. Investigating how this complex interacts with the silencing machinery promises to move the field forward and will be an exciting area for future studies.

\section{ACKNOWLEDGMENTS}

The research from this laboratory is supported by the Max Planck Society, by grants from the Deutsche Forschungsgemeinschaft (DFG, FOR855, and the Gottfried Wilhelm Leibniz Program awarded to E.I.).

\section{REFERENCES}

Aukerman MJ, Sakai H. 2003. Regulation of flowering time and floral organ identity by a MicroRNA and its APETALA2-like target genes. Plant Cell 15: 2730-2741.

Bagga S, Bracht J, Hunter S, Massirer K, Holtz J, Eachus R, Pasquinelli AE. 2005. Regulation by let-7 and lin-4 miRNAs results in target mRNA degradation. Cell 122: 553-563.

Bartel DP. 2009. MicroRNAs: Target recognition and regulatory functions. Cell 136: 215-233.

Bazzini AA, Lee MT, Giraldez AJ. 2012. Ribosome profiling shows that miR-430 reduces translation before causing mRNA decay in zebrafish. Science 336: 233-237.

Behm-Ansmant I, Rehwinkel J, Doerks T, Stark A, Bork P, Izaurralde E. 2006. mRNA degradation by miRNAs and GW182 requires both CCR4:NOT deadenylase and DCP1:DCP2 decapping complexes. Genes Dev 20: 18851898.

Braun JE, Huntzinger E, Fauser M, Izaurralde E. 2011. GW182 proteins recruit cytoplasmic deadenylase complexes to miRNA targets. Mol Cell 44: 120-133.
Brodersen P, Sakvarelidze-Achard L, Bruun-Rasmussen M, Dunoyer P, Yamamoto YY, Sieburth L, Voinnet O. 2008. Widespread translational inhibition by plant miRNAs and siRNAs. Science 320: 1185-1190.

Chekulaeva M, Filipowicz W, Parker R. 2009. Multiple independent domains of dGW182 function in miRNA-mediated repression in Drosophila. RNA 15: 794-803.

Chekulaeva M, Mathys H, Zipprich JT, Attig J, Colic M, Parker R, Filipowicz W. 2011. miRNA repression involves GW182-mediated recruitment of CCR4-NOT through conserved W-containing motifs. Nat Struct Mol Biol 18: $1218-1226$.

Chen X. 2004. A microRNA as a translational repressor of APETALA2 in Arabidopsis flower development. Science 303: 2022-2025.

Chen CY, Shyu AB. 2011. Mechanisms of deadenylationdependent decay. RNA 2: 167-183.

Chen CY, Zheng D, Xia Z, Shyu AB. 2009. Ago-TNRC6 triggers microRNA-mediated decay by promoting two deadenylation steps. Nat Struct Mol Biol 16: 1160-1166.

Chu CY, Rana TM. 2006. Translation repression in human cells by microRNA-induced gene silencing requires RCK/p54. PLoS Biol 4: e210.

Cooke A, Prigge A, Wickens M. 2010. Translational repression by deadenylases. J Biol Chem 285: 28506-28513.

Davey NE, Van Roey K, Weatheritt RJ, Toedt G, Uyar B, Altenberg B, Budd A, Diella F, Dinkel H, Gibson TJ. 2012. Attributes of shirt linear motifs. Mol Biosyst 8: 268-281.

Derry MC, Yanagiya A, Martineau Y, Sonenberg N. 2006. Regulation of poly(A)-binding protein through PABPinteracting proteins. Cold Spring Harb Symp Quant Biol 71: 537-543.

Ding XC, Großhans H. 2009. Repression of C. elegans microRNA targets at the initiation level of translation requires GW182 proteins. EMBO J 28: 213-222.

Ding L, Spencer A, Morita K, Han M. 2005. The developmental timing regulator AIN-1 interacts with miRISCs and may target the argonaute protein ALG-1 to cytoplasmic P bodies in C. elegans. Mol Cell 19: 437-447.

Djuranovic S, Nahvi A, Green R. 2011. A parsimonious model for gene regulation by miRNAs. Science 331: 550-553.

Djuranovic S, Nahvi A, Green A. 2012. miRNA-mediated gene silencing by translational repression followed by mRNA deadenylation and decay. Science 336: 237-240.

Dugas DV, Bartel B. 2008. Sucrose induction of Arabidopsis miR398 represses two $\mathrm{Cu} / \mathrm{Zn}$ superoxide dismutases. Plant Mol Biol 67: 403-417.

Esteller M. 2011. Non-coding RNAs in human disease. Nat Rev Genet 12: 861-874.

Eulalio A, Rehwinkel J, Stricker M, Huntzinger E, Yang SF, Doerks T, Dorner S, Bork P, Boutros M, Izaurralde E. 2007a. Target-specific requirements for enhancers of decapping in miRNA-mediated gene silencing. Genes Dev 21: $2558-2570$.

Eulalio A, Behm-Ansmant I, Izaurralde E. 2007b. P bodies: at the crossroads of post-transcriptional pathways. Nat Rev Mol Cell Biol 8: 9-22.

Eulalio A, Behm-Ansmant I, Schweizer D, Izaurralde E. 2007c. P-body formation is a consequence, not the cause 
J.E. Braun et al.

of RNA-mediated gene silencing. Mol Cell Biol 27: 3970-3981.

Eulalio A, Huntzinger E, Izaurralde E. 2008. GW182 interaction with Argonaute is essential for miRNA-mediated translational repression and mRNA decay. Nat Struct Mol Biol 15: 346-353.

Eulalio A, Huntzinger E, Nishihara T, Rehwinkel J, Fauser M, Izaurralde E. 2009a. Deadenylation is a widespread effect of miRNA regulation. RNA 15: 21-32.

Eulalio A, Tritschler F, Izaurralde E. 2009b. The GW182 protein family in animal cells: New insights into domains required for miRNA mediated gene silencing. RNA 15:1433-1442.

Eulalio A, Helms S, Fritzsch C, Fauser M, Izaurralde E. 2009c. A C-terminal silencing domain in GW182 is essential for miRNA function. RNA 15: 1067-1077.

Eystathioy T, Chan EK, Tenenbaum SA, Keene JD, Griffith K, Fritzler MJ. 2002. A phosphorylated cytoplasmic autoantigen, GW182, associates with a unique population of human mRNAs within novel cytoplasmic speckles. Mol Biol Cell 13: 1338-1351.

Fabian MR, Mathonnet G, Sundermeier T, Mathys H, Zipprich JT, Svitkin YV, Rivas F, Jinek M, Wohlschlegel J, Doudna JA, et al. 2009. Mammalian miRNA RISC recruits CAF1 and PABP to affect PABP-dependent deadenylation. Mol Cell 35: 868-880.

Fabian MR, Cieplak MK, Frank F, Morita M, Green J, Srikumar T, Nagar B, Yamamoto T, Raught B, Duchaine TF et al. 2011. miRNA-mediated deadenylation is orchestrated by GW182 through two conserved motifs that interact with CCR4-NOT. Nat Struct Mol Biol 18: 1211-1217.

Fukaya T, Tomari Y. 2011. PABP is not essential for microRNA-mediated translational repression and deadenylation in vitro. EMBO J 30: 4998-5009.

Gandikota M, Birkenbihl RP, Höhmann S, Cardon GH, Saedler H, Huijser P. 2007. The miRNA156/157 recognition element in the $3^{\prime}$ UTR of the Arabidopsis SBP box gene SPL3 prevents early flowering by translational inhibition in seedlings. Plant J 49: 683-693.

Giraldez AJ, Mishima Y, Rihel J, Grocock RJ, Van Dongen S, Inoue K, Enright AJ, Schier AF. 2006. Zebrafish MiR-430 promotes deadenylation and clearance of maternal mRNAs. Science 312: 75-79.

Guo H, Ingolia NT, Weissman JS, Bartel DP. 2010. Mammalian microRNAs predominantly act to decrease target mRNA levels. Nature 466: 835-840.

Hendrickson DG, Hogan DJ, McCullough HL, Myers JW, Herschlag D, Ferrell JE, Brown PO. 2009. Concordant regulation of translation and mRNA abundance for hundreds of targets of a human microRNA. PLoS Biol 7: e1000238.

Humphreys DT, Westman BJ, Martin DI, Preiss T. 2005. MicroRNAs control translation initiation by inhibiting eukaryotic initiation factor $4 \mathrm{E} / \mathrm{cap}$ and poly(A) tail function. Proc Natl Acad Sci 102: 16961-16966.

Huntzinger E, Izaurralde E. 2011. Gene silencing by microRNAs: Contributions of translational repression and mRNA decay. Nat Rev Genet 12: 99-110.

Huntzinger E, Braun JE, Heimstädt S, Zekri L, Izaurralde E. 2010. Two PABPC-binding sites in GW182 proteins pro- mote miRNA-mediated gene silencing. $Е M B O J$ 29: 4146-4160.

Iwasaki S, Kawamata T, Tomari Y. 2009. Drosophila argonaute1 and argonaute2 employ distinct mechanisms for translational repression. Mol Cell 34: 58-67.

Jakymiw A, Lian S, Eystathioy T, Li S, Satoh M, Hamel JC, Fritzler MJ, Chan EK. 2005. Disruption of GW bodies impairs mammalian RNA interference. Nat Cell Biol 7: 1267-1274.

Jínek M, Doudna JA. 2009. A three-dimensional view of the molecular machinery of RNA interference. Nature 457: 405-412.

Jínek M, Fabian MR, Coyle SM, Sonenberg N, Doudna JA. 2010. Structural insights into the human GW182-PABC interaction in microRNA-mediated deadenylation. Nat Struct Mol Biol 17: 238-240.

Kozlov G, Safaee N, Rosenauer A, Gehring K. 2010. Structural basis of binding of P-body associated protein GW182 and Ataxin-2 by the MLLE domain of poly(A)binding protein. J Biol Chem 285: 13599-13606.

Krol J, Loedige I, Filipowicz W. 2010. The widespread regulation of microRNA biogenesis, function and decay. $\mathrm{Na}$ ture Rev Genet 11: 597-610.

Kuzuoglu-Öztürk D, Huntzinger E, Schmidt S, Izaurralde E. 2012. The Caenorhabditis elegans GW182 protein AIN-1 interacts with PAB-1 and subunits of the PAN2-PAN3 and CCR4-NOT deadenylase complexes. Nucleic Acids Res doi: $10.1093 /$ nar/gks218.

Lazzaretti D, Tournier I, Izaurralde E. 2009. The C-terminal domains of human TNRC6A, B and C silence bound transcripts independently of the Argonaute proteins. RNA 15: 1059-1066.

Lian SL, Abadal GX, Pauley BA, Fritzler MJ, Chan EKL. 2009. The C-terminal half of human Ago2 binds to multiple GW-rich regions of GW182 and requires GW182 to mediate silencing. RNA 15: 804-813.

Lim LP, Lau NC, Garrett-Engele P, Grimson A, Schelter JM, Castle J, Bartel DP, Linsley PS, Johnson JM. 2005. Microarray analysis shows that some microRNAs downregulate large numbers of target mRNAs. Nature 433: 769-773.

Liu J, Rivas FV, Wohlschlegel J, Yates JR III, Parker R, Hannon GJ. 2005. A role for the P-body component GW182 in microRNA function. Nat Cell Biol 7: 1261-1266.

Maroney PA, Yu Y, Fisher J, Nilsen TW. 2006. Evidence that microRNAs are associated with translating messenger RNAs in human cells. Nat Struct Mol Biol 13: 1102-1107.

Mathonnet G, Fabian MR, Svitkin YV, Parsyan A, Huck L, Murata T, Biffo S, Merrick WC, Darzynkiewicz E, Pillai RS, et al. 2007. MicroRNA inhibition of translation initiation in vitro by targeting the cap-binding complex eIF4F. Science 17: 1764-1767.

Meister G, Landthaler M, Peters L, Chen PY, Urlaub H, Luhrmann R, Tuschl T. 2005. Identification of novel argonaute-associated proteins. Curr Biol 15: 2149-2155.

Mishima Y, Giraldez AJ, Takeda Y, Fujiwara T, Sakamoto H, Schier AF, Inoue K. 2006. Differential regulation of germline mRNAs in soma and germ cells by zebrafish miR430. Curr Biol 16: 2135-2142.

Mishima Y, Fukao A, Kishimoto T, Sakamoto H, Fujiwara T, Inoue K. 2012. Translational inhibition by deadenylation-independent mechanisms is central to microRNA- 
mediated silencing in zebrafish. Proc Natl Acad Sci doi: 10.1073/pnas.1113350109.

Nissan T, Rajyaguru P, She M, Song H, Parker R. 2010. Decapping activators in Saccharomyces cerevisiae act by multiple mechanisms. Mol Cell 39: 773-783.

Nottrott S, Simard MJ, Richter JD. 2006. Human let-7a miRNA blocks protein production on actively translating polyribosomes. Nature Struct Mol Biol 13: 1108-1114.

Olsen PH, Ambros V. 1999. The lin-4 regulatory RNA controls developmental timing in Caenorhabditis elegans by blocking LIN-14 protein synthesis after the initiation of translation. Dev Biol 216: 671-680.

Pasquinelli AE. 2012. A team effort blocks the ribosome in its tracks. Nature Struct Mol Biol 19: 133-134.

Petersen CP, Bordeleau ME, Pelletier J, Sharp PA. 2006. Short RNAs supress translation after initiation in mammalian cells. Mol. Cell 21: 533-542.

Piao X, Zhang X, Wu L, Belasco JG. 2010. CCR4-NOT deadenylates mRNA associated with RNA-induced silencing complexes in human cells. Mol Cell Biol 30: 1486-1494.

Pillai RS, Bhattacharyya SN, Artus CG, Zoller T, Cougot N, Basyuk E, Bertrand E, Filipowicz W. 2005. Inhibition of translational initiation by Let-7 MicroRNA in human cells. Science 309: 1573-1576.

Rehwinkel J, Behm-Ansmant I, Gatfield D, Izaurralde E. 2005. A crucial role for GW182 and the DCP1:DCP2 decapping complex in miRNA-mediated gene silencing. RNA 11: 1640-1647.

Rehwinkel J, Natalin P, Stark A, Brennecke J, Cohen SM, Izaurralde E. 2006. Genome-wide analysis of mRNAs regulated by Drosha and Argonaute proteins in Drosophila melanogaster. Mol Cell Biol 26: 2965-2975.

Sayed D, Abdellatif M. 2011. MicroRNAs in development and disease. Physiol Rev 91: 827-887.

Schmitter D, Filkowski J, Sewer A, Pillai RS, Oakeley EJ, Zavolan M, Svoboda P, Filipowicz W. 2006. Effects of Dicer and Argonaute down-regulation on mRNA levels in human HEK293 cells. Nucleic Acids Res 34: 48014815.

Seggerson K, Tang L, Moss EG. 2002. Two genetic circuits repress the Caenorhabditis elegans heterochronic gene lin-28 after translation initiation. Dev Biol 243: 215-225.

Siddiqui N, Mangus DA, Chang TC, Palermino JM, Shyu AB, Gehring K. 2007. Poly(A) nuclease interacts with the $\mathrm{C}$-terminal domain of polyadenylate-binding protein domain from poly(A)-binding protein. J Biol Chem 282: 25067-25075.

Su H, Meng S, Lu Y, Trombly MI, Chen J, Lin C, Turk A, Wang X. 2011. Mammalian hyperplastic discs homolog EDD regulates miRNA-mediated gene silencing. Mol Cell 43: $97-109$.

Takeda Y, Mishima Y, Fujiwara T, Sakamoto H, Inoue K. 2009. DAZL relieves miRNA-mediated repression of germline mRNAs by controlling poly(A) tail length in zebrafish. PLoS ONE 4: e7513.

Takimoto K, Wakiyama M, Yokoyama S. 2009. Mammalian GW182 contains multiple Argonaute binding sites and functions in microRNA-mediated translational repression. RNA 15: $1078-1089$.
Thermann R, Hentze MW. 2007. Drosophila miR2 induces pseudo-polysomes and inhibits translation initiation. Nature 447: 875-878.

Till S, Lejeune E, Thermann R, Bortfeld M, Hothorn M, Enderle D, Heinrich C, Hentze MW, Ladurner AG. 2007. A conserved motif in Argonaute-interacting proteins mediates functional interactions through the Argonaute PIWI domain. Nat Struct Mol Biol 14: 897-903.

Voinnet O. 2009. Origin, biogenesis, and activity of plant microRNA. Cell 136: 669-687.

Walters RW, Bradrick SS, Gromeier M. 2010. Poly(A)-binding protein modulates mRNA susceptibility to cap-dependent miRNA-mediated repression. RNA 16: 239250.

Wang B, Love TM, Call ME, Doench JG, Novina CD. 2006. Recapitulation of short RNA-directed translational gene silencing in vitro. Mol Cell 22: 553-560.

Wakiyama M, Takimoto K, Ohara O, Yokoyama S. 2007. Let7 microRNA-mediated mRNA deadenylation and translational repression in a mammalian cell-free system. Genes Dev 21: 1857-1862.

Wu L, Belasco JG. 2005. Micro-RNA regulation of the mammalian lin-28 gene during neuronal differentiation of embryonal carcinoma cells. Mol Cell Biol 25: 9198-9208.

Wu L, Fan J, Belasco JG. 2006. MicroRNAs direct rapid deadenylation of mRNA. Proc Natl Acad Sci 103: 40344039.

Wu E, Thivierge C, Flamand M, Mathonnet G, Vashisht AA, Wohlschlegel J, Fabian MR, Sonenberg N, Duchaine TF. 2010. Pervasive and cooperative deadenylation of $3^{\prime}$ UTRs by embryonic microRNA families. Mol Cell 40: 558-570.

Yang L, Wu G, Poethig RS. 2012. Mutations in the GWrepeat protein SUO reveal a developmental function for microRNA-mediated translational repression in Arabidopsis. Proc Natl Acad Sci 109: 315-320.

Yao B, Li S, Jung HM, Lian SL, Abadal GX, Han F, Fritzler MJ, Chan EK. 2011. Divergent GW182 functional domains in the regulation of translational silencing. Nucleic Acids Res 39: 2534-2547.

Zdanowicz A, Thermann R, Kowalska J, Jemielity J, Duncan K, Preiss T, Darzynkiewicz E, Hentze MW. 2009. Drosophila miR2 primarily targets the $\mathrm{m} 7 \mathrm{GpppN}$ cap structure for translational repression. Mol Cell 35: 881-888.

Zekri L, Huntzinger E, Heimstädt S, Izaurralde E. 2009. The silencing domain of GW182 interacts with PABPC1 to promote translational repression and degradation of miRNA targets and is required for target release. Mol Cell Biol 29: 6220-6231.

Zhang L, Ding L, Cheung TH, Dong M-Q, Che J, Sewell AK, Liu X, Yates JR, Han M. 2007. Systematic identification of C. elegans miRISC proteins, miRNAs, and mRNA targets by their interactions with GW182 proteins AIN-1 and AIN-2. Mol Cell 28: 598-613.

Zipprich JT, Bhattacharyya S, Mathys H, Filipowicz W. 2009. Importance of the $\mathrm{C}$-terminal domain of the human GW182 protein TNRC6C for translational repression. RNA 15: 781-793. 


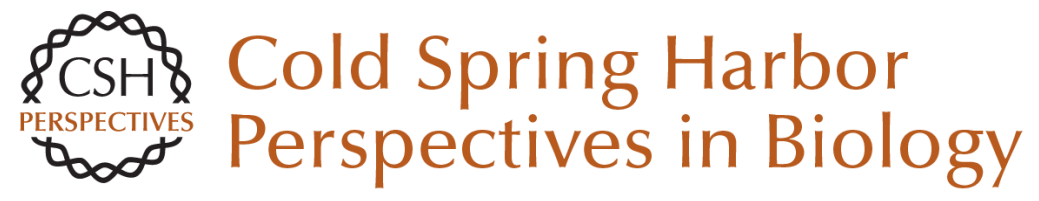

\title{
A Molecular Link between miRISCs and Deadenylases Provides New Insight into the Mechanism of Gene Silencing by MicroRNAs
}

\author{
Joerg E. Braun, Eric Huntzinger and Elisa Izaurralde
}

Cold Spring Harb Perspect Biol 2012; doi: 10.1101/cshperspect.a012328

\section{Subject Collection Protein Synthesis and Translational Control}

Tinkering with Translation: Protein Synthesis in

Virus-Infected Cells

Derek Walsh, Michael B. Mathews and lan Mohr

Translational Control in Cancer Etiology

Davide Ruggero

A Molecular Link between miRISCs and Deadenylases Provides New Insight into the Mechanism of Gene Silencing by MicroRNAs Joerg E. Braun, Eric Huntzinger and Elisa Izaurralde

Imaging Translation in Single Cells Using Fluorescent Microscopy

Jeffrey A. Chao, Young J. Yoon and Robert $H$. Singer

mRNA Localization and Translational Control in Drosophila Oogenesis Paul Lasko

P-Bodies and Stress Granules: Possible Roles in the Control of Translation and mRNA Degradation Carolyn J. Decker and Roy Parker

Protein Secretion and the Endoplasmic Reticulum Adam M. Benham
Toward a Genome-Wide Landscape of

Translational Control

Ola Larsson, Bin Tian and Nahum Sonenberg

The Current Status of Vertebrate Cellular mRNA

IRESs

Richard J. Jackson

Principles of Translational Control: An Overview John W.B. Hershey, Nahum Sonenberg and Michael B. Mathews

Regulation of mRNA Translation by Signaling Pathways

Philippe P. Roux and Ivan Topisirovic

The Mechanism of Eukaryotic Translation Initiation: New Insights and Challenges Alan G. Hinnebusch and Jon R. Lorsch

Single-Molecule Analysis of Translational Dynamics Alexey Petrov, Jin Chen, Seán O'Leary, et al.

Cytoplasmic RNA-Binding Proteins and the Control of Complex Brain Function Jennifer C. Darnell and Joel D. Richter

For additional articles in this collection, see http://cshperspectives.cshlp.org/cgi/collection/

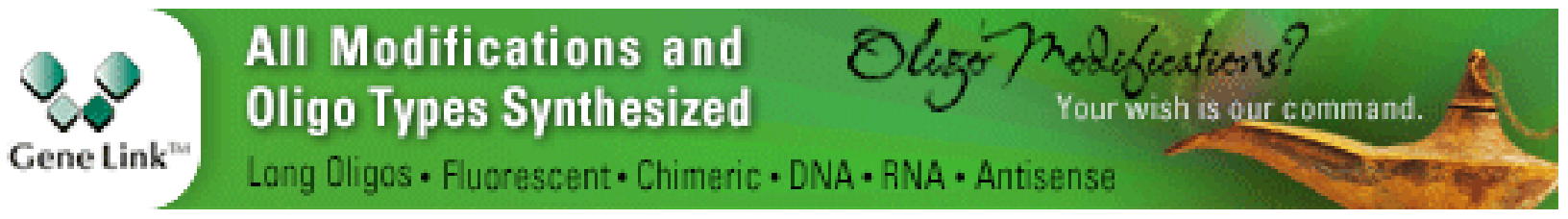




\section{From Cis-Regulatory Elements to Complex RNPs and Back}

Fátima Gebauer, Thomas Preiss and Matthias W. Hentze
The Elongation, Termination, and Recycling Phases of Translation in Eukaryotes

Thomas E. Dever and Rachel Green

For additional articles in this collection, see http://cshperspectives.cshlp.org/cgi/collection/

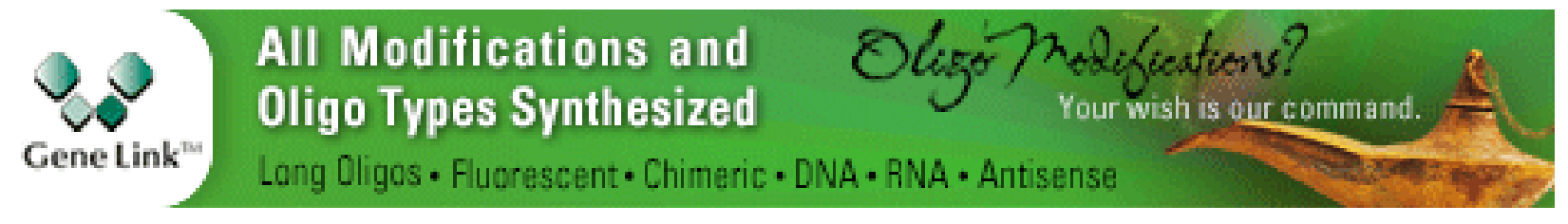

Copyright @ 2012 Cold Spring Harbor Laboratory Press; all rights reserved 